\title{
Correction to: Iris Identification in 3D
}

Fernand Cohen, Sowrirajan Sowmithran, and Chenxi Li

\section{Correction to: \\ Chapter "Iris Identification in 3D" \\ in: M. Felsberg et al. (Eds.): Image Analysis, LNCS 11482, https://doi.org/10.1007/978-3-030-20205-7_27}

The chapter "Iris Identification in 3D" by Fernand Cohen, Sowrirajan Sowmithran, and Chenxi Li (pp. 324-335) was not presented during the Scandinavian Conference on Image Analysis (SCIA) 2019. SCIA is embraced by IAPR and following the IAPR policy, only papers presented at the respective conference should be included in the proceedings. Thus, the chapter by Cohen et al. is not officially part of the SCIA conference proceedings LNCS 11482. Unfortunately, at the time of production of LNCS 11482, this fact was not known and therefore the manuscript is erroneously available for download within the proceedings. 